\title{
Una aproximación a las condiciones laborales de trabajo social: un insumo para el debate en la actualidad del ejercicio profesional ${ }^{*}$
}

\author{
Paula Vidal Molina**
}

\begin{abstract}
RESUMEN
Las condiciones laborales en que se desempeñan las profesiones en general han sufrido mutaciones. Ello ha afectado también a la profesión del Trabajo Social Chileno. Este estudio, cuya metodología priorizó una mirada cuantitativa, devela algunos de los cambios sufridos a nivel de las formas de contrato, el espacio profesional, la pluriempleabilidad y los salarios, entre otros, hacen creer que nos enfrentamos a una situación de creciente precarización. El reflexionar acerca de esos cambios ayudará a identificar tensiones de la práctica y los desafíos para la profesión en relación al momento actual en que Estado y modelo económico se entrelazan.
\end{abstract}

Palabras Clave: Neoliberalismo / Trabajo Social / Condiciones Laborales Chilenas / Precariedad.

\section{An approach to the working conditions of social work: food for thought and debate about the present state of affairs in the professional practice}

\begin{abstract}
The working conditions in which professions generally evolve have undergone changes. It has also affected the profession of the Chilean Social Work. This study, whose methodology prioritized a quantitative view, reveals some of the changes undergone concerning the forms of contract, the professional space, the multi-employbility and the wages, among others, which make us think that we are facing a situation of increasing deprivation. Reflecting about those changes will help us identify tension of the practice and the challenges for the profession in relation to the present moment at which State and economic model become overlapped.
\end{abstract}

Key words: neoliberalism / Social Work / Chilean Working Conditions / Deprivation

* Este artículo fue presentado originalmente con el nombre correspondiente a "condiciones laborales del trabajo social en chile: un insumo para el debate en la actualidad del ejercicio profesional"

** Chilena. Trabajadora Social. Magíster en Antropología y Desarrollo, Universidad de Chile. Correo electrónico: pvidal71@yahoo.com 


\section{Introducción}

Sin duda las condiciones laborales de los trabajadores han cambiado en los últimos decenios, la precarización y vulnerabilidad son parte de la vida de la mayoría y parece ser que ya nadie se asombra con esta incertidumbre, "se ha aprendido a vivir con ella". Lo contrario se ha constituido en lo "extra-ordinario". En ese sentido, Antunes parece seguir acertando al defender la tesis de que "la sociedad del capital y su ley del valor necesitan cada vez menos del trabajo estable y cada vez más de las diversificadas formas de trabajo parcial o part -time, mercerizado, que son, en escala creciente, parte constitutiva del proceso de producción capitalista" (Antunes 1999:10), debido a la vigencia de este panorama en la primera década del siglo XXI.

En esta situación, ¿quién se ha preguntado por la relación existente entre las condiciones laborales en que los trabajadores sociales realizan cotidianamente la intervención social y las posibilidades que abre o cierra -la intervención en lo social- en el horizonte de la defensa y lucha por los derechos sociales de la población "destinataria" o como acostumbramos llamar "población objetivo 1"? Pregunta que es posible derivar desde la preocupación por las condiciones laborales, que han mostrado escritos argentinos ${ }^{2}$, brasileros, reclamos españoles ${ }^{3}$ o evidencias chilenas ${ }^{4}$, los que no dejan de pronunciarse

1 Poco tiene de extraño el hecho de hurgar en el origen de las palabras como aquel primer paso para descubrir significados impensados para muchos de nosotros y potencialidades de éstas. Objetivo, vale la pena recordar, deriva del participio pasado latino jectum - variante de Jartum, que significa lanzado, colocado, puestoen composición con el prefijo latino ob cuyo significado es delante de, por lo tanto es lo que es lanzado, puesto delante de un subjectum, un objeto, hecho sujeto también, es decir, un sujeto lanzado en frente de un otro sujeto. En ese sentido, para ambos sujetos el otro sujeto se le es puesto enfrente.

2 Ver Cardemartori, Campos y Seiffer en "Condiciones de trabajo de los trabajadores sociales. Hacia un proyecto profesional crítico". Editorial Espacio, Buenos Aires, 2007.

3 Un estudio realizado en Aragón, España, acerca del perfil de los Trabajadores Sociales da cuenta además de la importante presencia de mujeres (el 92,77\% de las personas colegiadas son mujeres), de que el ejercicio profesional es realizado mayoritariamente en los servicios sociales (el 63,79\%), especialmente caracterizado por estar centrado en la función de atención directa (el 91,50\%), posee un rasgo distintivo en Aragón que es la situación de desempleo en que se encuentran muchos colegiados/as (el $44,40 \%$ se encuentra en situación de desempleo). Ver mayores antecedentes en www.tesisenxarxa.net y otro tipo de evidencia se ubica en Diagonal.web, periódico quincenal de actualidad crítica español, ubicado en www// diagonalperiodico.net/article2470.html

4 Interesante investigación es realizada por Javier Barría, Asistente Social, acerca del Síndrome de Burnout en asistentes sociales del Servicio Nacional de Menores de 
frente a ello, en algunos casos solo para constatar y en otras de una manera reflexiva, penetrante y política, que invitan -claramente- a plantearse la necesidad de profundizar este tema en Chile.

Lo anterior, si bien puede cobrar relevancia desde un interés institucional-académico restringido a la preocupación de la formación y pertinencia de los "formados" para la oferta laboral existente en el país en el corto, mediano o largo plazo, o desde los profesionales que se encuentran en el espacio laboral y que -en muchos casos- no les queda mas que padecer tal situación, creemos que su relevancia especialmente se configura en una mirada, un abordaje que ponga como fin el desentrañar, develar, las determinaciones y condicionamientos concretos en que se juega el trabajador social en su praxis, acción ${ }^{5}$, cuyo horizonte abre, despliega no solo el abanico de posibi-

la Región Metropolitana de Chile, 2002, en que sus hallazgos dan cuenta de que condiciones laborales con "contrato a plazo fijo y el sistema de contrata a honorarios (sin imposiciones previsionales ni de salud) también está asociado a la proclividad y presencia del Burnout, lo cual se constituye en una fuente constante de presión de pérdida del empleo (...) que el sistema contractual del Sename de tener contratos a honorarios y a plazo fijo afecta la vida laboral del funcionario, en la medida que está sujeto no a las demandas del trabajo sino del mercado (...)", se suman a los hallazgos que la gran cantidad de horas destinadas para atención de público no compatibiliza con la salud del profesional, o la extensión de la jornada de trabajo la cual se extiende a más de 8 horas diarias, hace más proclive el desarrollo del síndrome de Burnout por parte del Asistente Social. Ello unido a que a más bajos ingresos o ingresos insuficientes se afecta principal y negativamente la motivación laboral. Ver investigación en www.ergonmia.cl/burnout_chile.pdf . Lo anterior también se evidencia, por ejemplo, en la demanda pública por mejoras en las condiciones laborales del sector de profesionales universitarios de los servicios de salud chilena -Fenpruss- donde los Asistentes Sociales poseen un rol fundamental. El año 2006 declaran "Hoy es una gran preocupación para nuestra organización que más del sesenta porciento de los profesionales de la salud se encuentren en calidad jurídica de contrata, situación que ha contribuido a generar condiciones de trabajo precarias, a inequidades aberrantes, lo que ha impedido que estos profesionales puedan promocionarse al interior de una real carrera funcionaria. Es necesario entonces abordar esta problemática y buscar una solución para estos trabajadores. Los porcentajes actuales de la planta y la contrata encubren otras cifras de las cuales no tenemos certezas. Se trata de funcionarios a contrata y otras formas de vinculación". Ver en www.archivochile.com/Chile_actual/01_ms

5 El uso común del término praxis está vinculado a un aspecto utilitario, pero este no posee forzosamente una conexión semántica con ello. A la vez, es interesante indagar en el concepto de praxis o acción ya que han sido conceptos que han nutrido, dado cuerpo, en diversos momentos de la historia del Servicio Social, a su quehacer, a modo de ejemplo no hay que olvidar, la preeminencia de este término en el periodo de la reconceptualización, vinculado a la transformación social. Al indagar en ese uso, nos encontramos con que el sustantivo praxis proviene, por un lado del verbo 
lidades que posee el sujeto para que este por sí mismo, sin ayuda de muletas -como diría el viejo Kant- pueda decidir lo mejor, salga de su minoría de edad, se autodetermine, sino que a la vez, en ese acto ambos -en algún sentido- puedan pronunciarse performativamente por una sociedad "democrática y más justa".

\section{Al hablar de la precariedad de las condiciones laborales de los tra-}

griego práttein que designa acción, actividad, realización, ejecutar, realizar alguna cosa por si mismo y por otro lado, de la raíz latina agere que significa actuar, hacer. La praxis en griego antiguo es por tanto una acción, "significa la acción de llevar algo a cabo, pero una acción que tiene su fin en si misma, y que no crea o produce un objeto ajeno a agente 0 a su actividad."(Sánchez, Adolfo2007:28).Traducción propia. Arendt, plantea que Platón fue el primero en introducir la división entre pensamiento y acción, entre los que saben y no actúan y los que actúan y no saben "Es cierto -y Platón, que había obtenido la palabra clave de su filosofía, la "idea", de las experiencias en la esfera de la fabricación, debió de ser el primero en observarlo- que la división entre conocer y hacer, tan extraña a la esfera de la acción, cuya validez y significación se destruyen en el momento en que pensamiento y acción se separan, es una experiencia cotidiana en la fabricación, cuyos procesos se dividen en dos partes: en primer lugar, captar la imagen o aspecto (eidos) del producto que se va hacer, y luego organizar los medios y comenzar la ejecución" (Arendt, H.; 1998: 245-246). Platón y Aristóteles son los máximos exponentes de esta separación y diferenciación entre teoría y praxis."La idea de que el hombre se hace a si mismo, y se eleva como ser humano justamente por su actividad práctica, de su trabajo, transformando el mundo material -idea que solo surgirá en la conciencia filosófica moderna- era, en general, ajena al pensamiento griego. Para este, el hombre se eleva exactamente por el camino inverso, esto es, por la liberación de toda actividad práctica material, y por tanto, aislando la teoría de la práctica." (Sánchez, Adolfo; 2007:37). En el Renacimiento, la conciencia filosófica de la praxis sufre un cambio radical respecto de la griega antigua. En esta nueva perspectiva el hombre deja de ser un animal teórico y pasa a ser un sujeto activo, creador, constructor y transformador del mundo, de la naturaleza. Por otro lado, praxis en Hegel "no es sino un momento del desarrollo lógico de la Idea y, por tanto, debe ser cancelada o superada para permitir el pasaje a un nivel superior; la praxis material debe ser superada con la mera actividad espiritual (...) la praxis es, definitivamente, teórica, ya que solo encuentra su fundamento, su verdadera naturaleza y su fin en el propio movimiento teórico del absoluto. (...) Hegel revela tanto en la Fenomenología como en la Lógica aspectos importantes de la praxis real, efectiva, humana." (Sánchez, Adolfo; 2007:87). En Marx, el término praxis se transforma en una categoría central, en cuanto a partir de ella gana sentido la actividad del hombre, su historia y conocimiento, emergiendo el sentido de su filosofía, como filosofía de la transformación del mundo, haciéndose alusión a la tesis $11^{\text {a }}$ sobre Feuerbach. "La relación entre teoría y praxis es para Marx teórica y práctica; práctica en la medida en que la teoría, como guía de acción. moldea la actividad del hombre, particularmente la actividad revolucionaria; teórica, en la medida en que esta relación es conciente" (Sánchez, Adolfo; 2007:109). Con este breve recorrido solo me interesa mostrar que este concepto remite a una unión entre pensamiento, teoría y acción, ejecución, práctica y no como lo ha entendido el sentido común, -y no solo el-como algo de "segunda categoría", en relación a la teoría. En ese sentido, me permito libremente usar este concepto para caracterizar el hacer del profesional, a modo circular: un actuar pensando y un conocer actuando para transformar. 
bajadores en general, difícilmente se puede pensar que los Trabajadores Sociales se excluyan de este fenómeno. Al contrario, se puede suponer que tanto aquellos pertenecientes al ámbito estatal, privado y tercer sector, sufren directa o indirectamente sus consecuencias.

Frente a lo enunciado, adscribimos a aquella hipótesis que plantea Yolanda Guerra cuando dice que las "transformaciones macro-sociales producen alteraciones en las demandas profesionales, en los espacios de intervención; modifican las expresiones de las cuestiones sociales - materia prima de la intervención del trabajador social; provocan una redefinición de los objetos de intervención; atribuyen nuevas funciones a la profesión y nuevos criterios para la adquisición de nuevas legitimidades. Lo que se pretende afirmar es que las alteraciones en el "mundo del trabajo", en la esfera del Estado, en las políticas sociales, establecen nuevas mediaciones que se expresan en las condiciones objetivas (materiales y espirituales) sobre las cuales se realiza la intervención y que condicionan las respuestas de los profesionales" (Guerra, Yolanda; 2000:1). Tenemos que el quehacer profesional es mediado por los cambios dados en la sociedad y la profesión no puede desconocer aquellos, ni enfrentarse de espaldas a estos para entender el tipo de intervención que gestará, reconociendo también la brecha que existe con aquel que se debe realizar.

Así, con la intensión de contribuir a develar este ámbito en Chile, se hace este primer acercamiento a las condiciones laborales de un grupo de Trabajadores Sociales egresados de la Universidad Católica Silva Henríquez, quien a su haber posee ya en sus registros aproximadamente 850 egresados desde el año 1998 en adelante. Lo anterior, es debido a que es la primera universidad privada pionera en la formación de Trabajadores Sociales en una institución de esas características en Chile, con un cuerpo docente estable y con formación de postgrado, a la vez la escuela de Trabajo Social se encuentra permanentemente preocupada de reflexionar acerca de la formación de sus estudiantes en función de aportar profesionales cuyo horizonte ético sea de mejorar las condiciones de justicia social, solidaridad y equidad.

\section{Metodología}

El estudio presenta un diseño no experimental, incorporando una dimensión cuantitativa y una cualitativa En primera instancia sirvió de guía para el estudio el cuestionario realizado por Cademartori, 
Campos y Seiffer (2007). Sin embargo se realizaron algunas modificaciones, a fin de hacerlo pertinente al grupo de estudio chileno y a los fines buscados. Sus resultados fueron procesados con el programa de análisis computacional SPSS. El ámbito cualitativo fue recogido en una parte del cuestionario en que se plantearon preguntas abiertas en la que cada persona podría exponer y explicar sus puntos de vista. A este apartado se le aplicó un análisis de contenido. Ambas dimensiones del cuestionario permiten complementar los datos obtenidos.

El instrumento de recolección de datos fue enviado por correo electrónico a la totalidad de los profesionales pertenecientes a la Red de Egresados de Trabajo Social de la Universidad Católica Silva Henríquez, quienes bordean 420 personas, de un total de 850 egresados, es decir, el $50 \%$ de la totalidad de titulados. Se recibieron 50 cuestionarios con sus respectivas respuestas. La muestra fue no probabilística, y el perfil de los encuestados respondió a la posibilidad de que respondieran el cuestionario. Los datos se recogieron entre los meses de octubre y noviembre de 2007.

\section{Algunos antecedentes contextuales}

La hegemonía neoliberal es el telón de fondo de los cambios experimentados en nuestra sociedad, que ha sobrevivido en medio de múltiples crisis y reorganizaciones a lo largo de su historia. En ello, el Estado ha jugado un rol importante, ya que los capitalistas encuentran mayores riesgos para sus intereses si no cuentan con esa institucionalidad que los cubra, pero igualmente pueden funcionar sin este, ya que siempre han generado formas de protegerse en ambientes que no necesariamente aceptan sus reglas para hacer negocios. Pero "la condición preferida para la actividad capitalista es un Estado burgués en que instituciones de mercado y reglas contractuales (incluyendo las del contrato de trabajo) sean legalmente garantizadas y en que se creen estructuras de regulación para contener conflictos de clases" (Harvey 2005: 80).

El neoliberalismo sólo después de la crisis general de sobreacumulación de los años '70 vino a tornarse como una alternativa al Keynesianismo y a otras estructuras centrales del Estado en la formulación de políticas. Es de consenso que esta crisis desencadenó una serie de cambios a nivel del capital y del trabajo. Chile fue el primer país que experimentó el proyecto de construcción neoliberal 
del Estado. Luego en el año 1979 Inglaterra inicia su camino por la misma senda y en pocos años la doctrina neoliberal domina la política de todo el mundo, doctrina que, entre otras cosas, incorpora los activos de propiedad de los Estados o aquellos destinados al uso de la población en general, al mercado con el fin de invertir, valorizarlos y especular con ellos. Activos como la tierra, los bosques, las aguas pasan a ser vendidos como si fuesen mercancías de las empresas privadas. (Harvey, 2005)

En el actual contexto de la mundialización de la economía, el capital financiero adquiere preponderancia, grandes grupos industriales transnacionales se articulan al mundo de las finanzas, ya que tienen como soporte las instituciones financieras que pasan a operar con el capital que rinde intereses. Estos grupos industriales asumen formas cada vez más centralizadas y concentradas del capital industrial. "Las empresas industriales se asocian a las instituciones financieras (bancos, compañias de seguros, fondos de pensión, sociedades financieras de inversión colectiva y fondos mutuos), que pasan a comandar el conjunto de acumulación, configurando un modo específico de dominación social y política del capitalismo, con el soporte de los Estados Nacionales" (Iamamoto, 2007:108) ${ }^{6}$ En un mercado mundial unificado, se impulsa la tendencia a la homogeneización no sólo de los circuitos del capital, sino también de los modos de dominación ideológica y de los objetos de consumo, pero sustentada en la heterogeneidad y desigualdad de las economías nacionales (Iamamoto, 2007).

Es sabido que en paralelo a este proceso, en los países del capitalismo avanzado, en la época de los años '80, ocurrieron profundas transformaciones en el ámbito del trabajo: sus formas de inserción en la estructura productiva y en las formas de representatividad sindical y política, que afectaron su forma de ser (Antunes, 1999). Los centros de trabajo cuya base se anclaba en el sistema fordista modificaron sus estrategias de contratación, la organización del trabajo y atribución de tareas, el uso del tiempo y el sistema de remuneraciones, removiendo el sello de certeza, propio del patrón de la organización científica del Trabajo. Esta tendencia venía desarrollándose desde antes de la crisis de los años '70, pero a través de experiencias dirigidas a modificar en algunos puntos la rigidez del fordismo y el taylorismo se fue dando paso a aquello que en los años ` 80 y

6 Traducción de la autora. 
'90 el modelo japonés fue imponiendo, el toyotismo, influenciando enormemente a los países del primer mundo y luego al resto de los países. Este proceso trajo como consecuencia en el ámbito de los derechos del trabajo la desregulación de ellos, su flexibilización, por lo que las conquistas históricas de derechos por parte de los trabajadores fueron sustituidos o eliminados del mundo de la producción (Antunes, 1999).

Los nuevos modos de incorporación del trabajo a la producción ha ido mostrando cómo en este proceso de transformación el deterioro del trabajo ha sido un rasgo permanente, que hasta la fecha no se ha revertido, lo cual se atribuye al fuerte protagonismo que ha tenido el mercado en la fijación de éstas condiciones. Se reconoce que en el ámbito del trabajo existe una importante transformación, pero lejos de desaparecer, la figura del trabajo asalariado persiste, aunque adoptando formas cada vez más heterogéneas; "la sociedad del capital y su ley del valor necesitan cada vez menos del trabajo estable y cada vez más de las diversificadas formas del trabajo parcial o part time, tercerizado, que son, en escala creciente, parte constitutiva del proceso de producción capitalista" (Antúnez 1999:14).

El fenómeno de la globalización, la mundialización financiera, requiere de algunas condiciones para que se genere, y para ello fueron fundamentales los cambios que permitieron la apertura a los flujos financieros y de mercancías, la disminución o disolución del Estado en sus funciones económicas y sociales, y la flexibilización del mercado del trabajo. Este conjunto de cambios, en sintonía con los lineamientos del llamado "Consenso de Washington", ha generado una serie de desajustes que impactaron en diversos procesos sociales "La mundialización financiera bajo sus distintas vías de efectivización unifica, dentro de un mismo movimiento, procesos que vienen siendo tratados por los intelectuales como si fuesen aislados o autónomos: la "reforma" del Estado, tenida como específica de la arena política; la reestructuración productiva, referente a las actividades económicas empresariales y a la esfera del trabajo; la cuestión social, reducida a los llamados procesos de exclusión e integración social, generalmente circunscritos a dilemas de la eficacia de la gestión social, a la ideología neoliberal y concepciones postmodernas". (Iamamoto 2007:114)

De modo que los cambios dados a nivel mundial afectaron y afectan también las condiciones en que se desenvuelven hoy los trabajadores en Chile, ya que se configuraron diversas formas de ser 
trabajador dependiente, todas en coexistencia. Las condiciones hoy son menos estables. Un ejemplo de ello son los trabajadores subcontratados y otros que se encuentran al margen de toda protección y garantía laboral; la estabilidad de las condiciones de trabajo no es algo garantizado. En ese sentido, la inseguridad de los empleos y la variabilidad de las condiciones laborales constituyen parte del paisaje cotidiano, "Ello se debe, en buena parte, a que las condiciones de trabajo se vinculan a los resultados de la gestión de la empresa (participando los trabajadores en riesgos propios del capital), y también al resultado rentable $-y$, por tanto, aleatorio- que logra el propio trabajador en su desempeño; no siempre al tiempo que ocupa o al trabajo que realmente desarrolla." (Henríquez y Riquelme 2006:10).

Una reciente investigación de la Dirección del Trabajo de Chile, muestra que las condiciones laborales se deterioran en las empresas, pero que no sólo las condiciones y el empleo asalariado son inestables, sino también la sobrevivencia de las mismas unidades productivas se ha hecho muy incierta. En Chile tenemos que "La externalización de la producción y del trabajo es una característica del desarrollo actual, constituyéndose en una práctica frecuente de parte de las empresas. En los últimos veinte años se ha extendido en muchos países $y$, en ese contexto, Chile no es la excepción, tanto como estrategia desarrollada en forma abierta, promovida y celebrada por las empresas, como de modo soterrado, para disimular o evadir algún problema laboral, en muchos casos, y/o tributarios, en otros" (Henríquez y Riquelme 2006: 59)

A lo anterior, se anexa que uno de los factores que influyen en el deterioro de las condiciones laborales es que los acuerdos e intereses de los trabajadores aparecen segmentados y dispersos, donde se hace difícil la identificación de objetivos comunes, y con ello la unificación de fuerzas para la fijación de metas comunes para los trabajadores. Hoy no basta, como trabajador, poseer la más alta calificación para evadirse de este precario escenario. Debido a que por las precarias condiciones laborales que diagnostican profesionales con muy alta calificación, éstos prefieren emigrar a otros países para seguir sus trayectorias laborales, donde encuentran una cierta valoración que el mercado chileno no ofrece. Aparece así la desintegración de aquella relación generadora de certezas: a mayor calificación, mejor empleo, entiéndase mejores condiciones y mayor sueldo. 
El Estado no escapa a la dinámica anteriormente expuesta, ya que también ha desarrollado vías para externalizar acciones y contratar a funcionarios en precarias condiciones, unido a lo que se ha denominado "Modernización del Estado" y la "Reforma del Estado", que en una lógica que intenta poner criterios de administración, control de gestión orientado hacia los resultados y desempeño de funcionarios, como también criterios de calidad de servicios y atención al usuario, entre otros, va dejando en la indefensión a muchos trabajadores.

En este contexto, se hace necesario reconocer que estos cambios influyen en la profesión del Trabajo Social en Chile, donde se ha generado una alta explosión de escuelas técnicas y universitarias que ofrecen la formación de trabajo social y servicio social. Actualmente existe una oferta de 71 programas de formación en trabajo social que se imparten en universidades públicas como privadas y 59 programas que ofrecen los institutos técnicos y profesionales a nivel nacional, otorgando el título Asistente Social. El total de estudiantes, aproximadamente, para el año 2006, era de 24.400, donde 14.200 correspondían a estudiantes universitarios y 8.200 a institutos profesionales. No existen datos certeros acerca del número total de trabajadores sociales en Chile, pero se estima que llegan a unos 10.000 profesionales a nivel nacional (Ruz y Hernández 2007). Todo indica que a futuro el mercado de trabajo estará saturado, generando situaciones de cesantía y mayor precariedad al interior de la profesión.

\section{Análisis de datos}

\section{a) Perfil General}

Los datos encontrados muestran algunas características de las personas encuestadas que van a incidir en el análisis posterior.

La distribución por sexo de los casos analizados corresponde a un $80 \%$ de mujeres y un $20 \%$ de hombres, muy cercana a la matrícula total de trabajadores sociales en Chile, donde se alcanza un $85 \%$ de mujeres y un $15 \%$ de hombres, lo cual confirma la condición femenina de la profesión del Trabajo Social no solo en Chile, sino también en países como España, Argentina o Brasil.

La población es mayoritariamente joven, el 76\% se ubica entre los 
23 y 35 años, incluso el $40 \%$ de la muestra corresponde al tramo entre 23 y 30 años.

En relación al ingreso para la formación de una profesión como Trabajo Social, tenemos que un alto porcentaje, un $72 \%$ tuvo a esta carrera como primera opción de formación profesional frente a un $28 \%$ de las personas encuestadas que mencionan no haberla considerado inicialmente. Este último grupo optó por ella, principalmente porque evaluó positivamente su afinidad con temas de otras profesiones de las ciencias sociales como psicología o antropología. Otro argumento corresponde a la evaluación positiva del currículum y la existencia de campo laboral, lo que se constituyó en la motivación para ingresar.

En este ámbito es interesante incorporar la información cualitativa que refiere a los motivos por los cuales ingresan a estudiar Trabajo Social, puesto que ello da cuenta de la trama con que se ha tejido hasta ahora el ejercicio de la profesión por parte de cada entrevistado.

Uno de los principales motivos es que la mayoría otorga mucha importancia a la vocación de ayuda, al espíritu de servicio que poseen internamente, elemento que remite -en una primera mirada- a los trazos de identidad originarios de la profesión, vinculados a una versión laica del trabajo desarrollado desde la iglesia. El lenguaje utilizado está muy en sintonía con ello, un ejemplo es el siguiente: "Se es feliz ayudando a las personas con las dificultades que viven día a día (...) muchas veces con solo una orientación o palabra de aliento y señalar lo importante que son para su familia y otros, puedes robarle una sonrisa o lo mas importante es que tenía la solución cerca pero no la visualizaba"

Sin embargo, esta primera interpretación va adquiriendo complejidad en la medida que el discurso releva, en una dimensión de menor cuantía respecto de la primera, la importancia de la justicia social, la equidad, junto a la creencia en la capacidad de las personas para modificar sus condiciones de vida, es decir, en sus posibilidades de autonomía. Esto va configurando una motivación que se aleja de la visión tradicional religiosa, donde el paternalismo, la caridad es lo que prima, en una relación de verticalidad. Por lo tanto, la justicia social y potenciar la autonomía de las personas, parecen ser dos motivos que se conjugan con esta vocación de servicio. Lo anterior se expresa en el siguiente texto: "aportar a la construcción de un país más 
humano, justo, con paz y ayudar a las personas a mejorar su calidad de vida, proporcionándoles herramientas (...) potenciar, ser un facilitador".

Es posible apreciar dos elementos que concretan dimensiones de la justicia y la autonomía de las personas, como es la intención de romper con situaciones negativas o problemas que sufren las personas, unidos a la dimensión del contacto o trabajo directo ${ }^{7}$ con las personas más vulnerables, es decir, pareciera que la motivación se concretiza cuanto se trabaja directamente con los2 pobres", "marginados", "vulnerables", "excluidos" o alguna categoría que remita a una situación similar y en función de intentar solucionar algunos problemas o situaciones injustas que viven las personas: "El trabajo directo con personas, poder intervenir y tratar de solucionar en parte los problemas que afectan su vida (...) apoyar y acompañar situaciones de vida y mostrar caminos que lleven a intentar romper círculos de violencia, maltrato"

Es interesante notar que cobra muy baja relevancia, en el discurso, la motivación por apoyar, fomentar el cambio del orden social, esto es "ser agente de cambio social" o de la estructura social. El compromiso con el mundo popular, discurso propio del período de la reconceptualización, ha sido abandonado con el pasar del tiempo. A la vez, es notable la ausencia de la mención de las causas que generan las dificultades o situaciones difíciles que viven las personas y la manera en que esta profesión puede influir o modificar en algo este espacio. Por ejemplo, a través de la defensa de los Derechos Humanos, económicos, sociales-culturales, que corresponden a aspectos que no son explícitamente mencionados como motivaciones para la elección profesional.

Por último, un factor de menor peso en las respuestas, pero no por ello menos relevante a la hora de configurar un perfil profesional, es el de que algunos eligieron la profesión por elementos pragmáticos como es la existencia de campo laboral y el tipo de currículum que la profesión ofrecía, afín con otras profesiones del área de las ciencias sociales a las que no habrían podido ingresar.

7 Se sigue evidenciando esa dimensión que marcó en el origen al trabajo social chileno, su trabajo "en el abajo", pieza clave para la instalación de la reforma social moderna impulsada por el Estado Social de los años '20, ya que no era suficiente la promulgación tanto del Código del Trabajo, ni de la Constitución de 1925. Ver Illanes (2007). 
Esto es preocupante si es que consideramos que en esta elección existe un componente de frustración respecto de otra formación que se deseaba tener, quedando la elección por esta profesión en un lugar de relativa importancia, lo que podría tener consecuencias a la hora de ejercer profesionalmente.

Es pertinente discutir las motivaciones y la percepción que de estos actores acerca de la correspondencia entre éstas y lo que efectivamente pueden desarrollar laboralmente. Es tal sentido, encontramos que la mayoría siente la correspondencia entre lo que lo motivó a ser un trabajador social y lo que puede realizar, es decir, esta vocación de ayuda al servicio de los más desposeídos, excluidos, para solucionar los problemas entregándoles herramientas concretas para ello a las personas, sienten que es algo que pueden realizar y en lo que se sienten satisfechos: "Todos los días puedo dar una sonrisa, una palabra de aliento apuntando a solucionar en parte los problemas que afectan su vida (...), puedo apoyar procesos de mejoramiento, el trabajo en el terreno mismo apoyado desde la teoría"

Sin embargo, existe un grupo en el estudio que da cuenta de la relativa correspondencia, entre sus motivaciones y el ejercicio profesional, percibiendo que las instituciones burocráticas muchas veces limitan ello, pero también dan cuenta de que siempre las instituciones otorgan un margen en el cual el profesional puede maniobrar en función de sus motivaciones personales-profesionales: “No siempre se puede porque hay directrices e intereses institucionales que sirven de impedimento (...) a pesar de que la estructura determina en el ámbito laboral, es posible realizar algo de lo personal"

\section{Nivel de Estudios}

Respecto de la formación profesional actual del grupo estudiado, cabe destacar que un $76 \%$ de ellos, posee estudios posteriores a la titulación de trabajador social. Ello demuestra la importancia que tiene la permanente capacitación-especialización, a pesar de los costos económicos que esto implica. Se denota se sincronización con las exigencias del mercado: permanente calificación.

En un 66\% quienes siguen estudios posteriores a su titulación, independientemente del nivel de estudios y capacitación, se autofinancian los estudios. Luego en orden de importancia, un 26\% de los estudios de los encuestados, son financiados por el empleador; 
mientras que el 2\% de encuestados, cuenta con financiamiento compartido, esto es por parte del empleador y el propio trabajador. Este porcentaje puede ser considerado bajo si lo analizamos a la luz del impacto negativo sufren los ingresos del hogar.

Una vía de financiamiento de bajo impacto son las becas y pasantías, que sólo apoyan al 6\% de los miembros del estudio.

Del total que ha seguido estudios posteriores a su titulación, un $33 \%$ ha optado por una formación de menos de 6 meses de duración y un $21 \%$ por cursos de hasta 12 meses de duración, tal vez fuertemente orientados a las exigencias de la labor que realizan en sus respectivas instituciones. Por otro lado, tenemos que un $40 \%$ de los miembros del estudio, ha priorizado el estudio de diplomados, tal vez ello confirma la tendencia a apoyar las áreas en que se desarrollan laboralmente. Al momento del estudio no había estudiantes de magíster, no obstante, un 6\% cursaba doctorados. Es sabido que la formación de postgrado es fundamental para generar una masa crítica al interior de una categoría profesional; por lo mismo es imprescindible fomentarla al interior de la profesión. Sin embargo, ello se ve dificultado por las condiciones de financiamiento existentes, que como ya revisamos, están fuertemente centradas en los ingresos individuales (ya sea a través de créditos o del salario con que se cuenta) que tiene consecuencias negativas a nivel de los ingresos del Hogar.

\section{Relaciones de dependencia laboral}

Transitando desde el ámbito de caracterización de la muestra, nos adentramos en un espacio analítico de los elementos que van configurando las condiciones laborales de los Trabajadores Sociales. En este ámbito encontramos que el $24 \%$ de las personas encuestadas posee más de 9 años de ejercicio profesional; por lo tanto, representa a la primera generación de titulados ${ }^{8}$. Mientras que el $26 \%$ posee entre 3 y 9 años de ejercicio profesional, lo que sumado al primer valor, significa que el $50 \%$ de la muestra cuenta con una experiencia profesional de entre 3 a más de 9 años. Entretanto, el 20\% de quienes respondieron el cuestionario, posee entre 1 a 3 años de experiencia profesional y un 30\% entre 1 a 12 meses en la profesión.

8 Cabe destacar que este estudio fue realizado durante el año 2007. 
Cerca del 90\% de la muestra está empleado y cumpliendo funciones de Trabajador Social, lo que sería positivo especialmente para la escuela, y la pertinencia de la formación profesional impartida con la oferta de empleos. Ello en tanto se asume este dato como un indicador de inserción en el mercado laboral en el área del Trabajo Social.

Por otra parte tenemos que el $12 \%$ de quienes respondieron el cuestionario, no se encuentra empleado como Trabajador Social. Un 67\% de ese total, se desempeña en alguna otra función, y un 33\%, se encuentra cesante y en búsqueda de empleo como trabajador social. Interesante sería profundizar en las causas de la cesantía existente en estos profesionales, pero que no abordaremos en este estudio.

Desde otra perspectiva sostenemos que este estudio confirma la tendencia histórica de inserción de Trabajo Social en la esfera pública/estatal, ya que un 58\% de los encuestados, se desarrollan específicamente en municipalidades y sus dependencias, que corresponden a instancias ejecutoras de políticas sociales y por otro lado, dependencias ministeriales vinculadas a políticas sociales. Le sigue en importancia el empleo en el tercer sector (entiéndase ONGs, Corporaciones, Fundaciones, instancias de Educación técnica o superior, entidades filantrópicas, entre otras) que representan el 26\% de los encuestados. Es sabido que este sector está fuertemente financiado con dineros estatales a través de proyectos y programas que responden a políticas, líneas de trabajo y objetivos definidos por el nivel gubernamental, por lo que se habla de externalización de las funciones del estado en estas instituciones. Además aparecen fundaciones vinculadas a empresas privadas y que viabilizan la "responsabilidad social de la empresa". Por último, un 16\% de los miembros del estudio, se desempeñan el sector privado o empresas. Tal tendencia es similar en países como Argentina y Brasil.

\section{Valoración y delimitación de acciones al interior del Espacio Laboral}

Es interesante hacer notar que la mayoría de los encuestados -un 91\%- dan cuenta de la claridad por parte de la institución, respecto de las funciones que deben realizar como trabajadores sociales, aspecto vital para responder con pertinencia y calidad. Sin embargo, este indicador además de ser interpretado en un sentido positivo, 
presenta también un lado negativo, en tanto que la excesiva claridad de las funciones que debe realizar el trabajador social les puede imponer límites muy rígidos en sus funciones atentando contra los profesionales que desean incursionar en áreas de otro orden o innovar en sus prácticas.

Respecto de la jefatura inmediata, el $66 \%$ de los encuestados da cuenta de que éste rol es desempeñado por un profesional distinto del trabajo social. Este elemento es significativo en la medida en que las tareas que desarrollan los miembros del estudio, en su gran mayoría están dentro del área de políticas sociales y de desarrollo, áreas que históricamente han competido al trabajo social, aún cuando en la actualidad están siendo abordadas por otras profesiones. La relevancia del tipo de profesiones en cargos de jefaturas radica en que se va conformando la trama de legitimidad y poder de una profesión. Lo anterior no constituye una negación de la interdisciplinariedad, ni de equipos. Al contrario, es sabido de la necesidad de esto para la realización de las tareas que implica el trabajo en el mundo de lo social.

El que el espacio institucional-laboral genere una sensación de valoración positiva del quehacer como trabajador social es un aspecto muy positivo para los encuestados. El $72 \%$ de ellos se siente valorado y reconocido institucionalmente y un $28 \%$ considera que su quehacer profesional no es valorado. Como sabemos, el reconocimiento de ello es un elemento que aumenta la satisfacción con el trabajo aunque éste posea otros factores negativos como bajos salarios o precarios contratos.

Es preciso asumir que el mercado de trabajo se encuentra en permanente cambio. Esto se materializa en la reestructuración de los procesos de producción en la empresa, como vimos al comienzo con el cambio en los procesos de producción de un fordismo al toyotismo y por la modernización o reforma del estado. Estas transformaciones han influido en cambios al interior de los cargos y funciones o tareas asignadas y ejercidas por los profesionales. No obstante ello, en la profesión esos cambios no se han materializado significativamente, manteniéndose a grandes rasgos las principales funciones que han desarrollado históricamente los Asistentes Sociales. El estudio muestra que un $26 \%$ desarrolla funciones directas con personas de orden asistencial, un $25 \%$ se dedica a tareas vinculadas con la gestión, formulación y coordinación de planes y programas 
institucionales, un $20 \%$ se desenvuelve en funciones directas con organizaciones sociales y grupos territoriales. Mientras que un $11 \%$ de quienes respondieron el cuestionario, desarrollan funciones relacionadas con ámbitos de atención clínica-preventiva. Un $8 \%$ se desempeña en cargos de jefaturas institucionales, en cualquiera de sus dependencias, cabe destacar que dichos cargos no siempre dependen exclusivamente de competencias profesionales. Y por último, un área que se viene desarrollando, es el de capacitación y asesorías externas a grupos e instituciones, con un 5\% de los miembros del estudio dedicados a eso. ${ }^{9}$

\section{Nivel de Salarios}

El estudio da cuenta que el nivel de salario recibido por los profesionales se desglosa de la siguiente manera en pesos chilenos y dólares americanos:

- El $20 \%$ de los miembros del estudio recibe una remuneración bruta que oscila entre los 401.000 y los 500.000 pesos chilenos, lo que en dólares equivale a un monto que fluctúa entre los 800 y los 1.000. Sin embargo, a este monto se le descuenta el 20\% correspondiente a los gastos por conceptos de seguro de salud y de jubilación, recibiendo el trabajador un salario líquido va desde 320.000 a 400.000 pesos chilenos, equivalente a unos 640 a 800 dólares.

- Un $18 \%$ de los encuestados recibe un salario que oscila entre los 751.000 a 900.000 pesos chilenos bruto, equivalentes a 1.500 y 1.800 dólares, pero que al descontar los costos por conceptos de protección de salud y jubilación, aproximadamente el 20\% del ingreso bruto, el salario líquido recibido por el trabajador oscila entre 600.000 a 720.000 pesos, equivalentes a 1.200 y 1.440 dólares.

9 En un estudio citado por Omar Ruz y Jeannette Hernández (2007), dan cuenta que en Chile las funciones de los trabajadores sociales se orientan a lo siguiente: un "39\% de los trabajadores sociales realiza tareas de intervención de casos, grupos, comunidad, orientación, apoyo y gestión social en general; un 23,3\% se encuentra en el nivel de coordinación, que comprende: planificación, asesoría, diagnóstico, supervisión, con niveles de jefatura de programas, elaboración y diseño de proyectos y políticas sociales. El resto de los porcentajes esta distribuido entre funciones de bienestar y docencia" (Ruz, Omar, Hernández, Jeannette 2007: 126-127). 
- El $16 \%$ recibe un salario bruto que oscila entre los $501.000 \mathrm{y}$ los 600.000 pesos, es decir, entre 1.000 y 1.200 dólares, pero que descontado los seguros por conceptos de jubilación y protección de salud, el salario líquido recibido va desde 400.000 y 480.000 pesos chilenos, equivalente a un monto entre 800 y 960 dólares.

- Un $14 \%$ de los encuestados en el estudio recibe un salario bruto que va entre los 601.000 y 750.000 pesos chilenos, es decir, entre unos 1.200 y 1.500 dólares, pero que descontando los seguros correspondientes, la remuneración líquida recibida por el trabajador varía entre los 480.000 y los 600.000 pesos chilenos, unos 960 y 1.200 dólares.

- El 20\% de los trabajadores sociales participantes en este estudio, no gana más de 400.000 pesos chilenos bruto como salario, equivalente a unos 800 dólares y que descontado el $20 \%$ de los seguros antes mencionados, recibe una remuneración líquida de 320.000 pesos chilenos, es decir, unos 640 dólares.

- Un $12 \%$ recibe un salario bruto superior a 900.000 pesos chilenos, es decir, unos 1.800 dólares y más, pero que descontado el 20\% por los seguros, la remuneración líquida recibida por el trabajador no desciende de 720.000 pesos chilenos, equivalente a no menos de 1.440 dólares.

Frente a estos datos es relevante hacer un análisis en función del sueldo mínimo existente en Chile, el cual corresponde a 144.000 pesos, es decir, unos 288 dólares. Si se considera que la línea de la pobreza se fijó para el año 2006 en 47.100 pesos, correspondiente al valor de la canasta de satisfacción de necesidades básicas per cápita (canasta que no ha variado en los últimos 20 años), tenemos que para una familia media de 3,9 miembros se debe recibir un ingreso mensual de 180.000 pesos chilenos, solo considerando alimentación, ya que al incluir gastos básicos de vivienda, transporte, vestuario, educación de los hijos e hijas, vestuario, cargos por salud y jubilación (AFP), el resultado sería un ingreso mínimo bruto de aproximadamente 312.000 pesos, lo que dista mucho de los 144.000 pesos correspondiente al sueldo mínimo bruto. ${ }^{10}$ Lo anterior es relevante ya que permite analizar los salarios percibidos por los trabajadores

10 Ver www.piensachile.com 
sociales en este estudio, donde un $40 \%$ de quienes respondieron el cuestionario, no recibe un salario líquido superior a 400.000 pesos chilenos, lo cual es preocupante si es que se tiene en cuenta que este salario puede ser el único percibido por el grupo familiar.

Si se le suma el $16 \%$ que recibe un salario superior, tenemos que en términos generales un $56 \%$ de los miembros del estudio no posee un ingreso superior a 480.000 pesos líquidos, es decir, 4 veces el sueldo mínimo que, como ya se dijo, no es representativo de los gastos reales en los que se incurre para vivir. Por lo mismo, si tomamos el valor aproximado que se necesitaría (312.000 pesos chilenos) tenemos que los 480.000 pesos no alcanzan a doblar esos ingresos básicos, por lo tanto, se podría suponer que los recursos utilizados para estudios posteriores a la titulación, coresponden a endeudamientos o a restricciones importantes en otros bienes materiales o inmateriales básicos. En ese sentido, cobra relevancia el apuntar a un trabajo gremial que pueda normar los ingresos económicos mínimos que deben recibir los trabajadores sociales por determinadas intervenciones ${ }^{11}$ para garantizar condiciones básicas de vida y de calidad del ejercicio profesional.

Los bajos salarios obligaría al profesional a desarrollar trabajos en paralelo, lo que podría atentar contra la intervención que realizada, tornándola mecanizada y descomprometida con principios que la profesión dice valorar y que no siempre están presentes en las políticas con que se ejecuta el trabajo.

El salario del 50\% de los encuestados, posee una función primordial en el hogar porque éste se presenta como el ingreso principal o único de la familia. En el otro 50\% se constituye en un ingreso complementario a los ingresos totales de la familia. Lo primero es preocupante si es que se considera la media de los ingresos percibidos por ellos y la conformación media de una familia chilena, de 3 a 4 personas, salario, por lo tanto insuficiente para el sustento familiar y que pone en el debate el tema de la pluriempleabilidad y su relación con la defensa de los derechos de los usuarios.

11 Es interesante reconocer el avance en este aspecto por parte del Servicio Social en Brasil quienes han elaborado, por parte del Conselho Federal de Serviço Social en el año 2001, una Tabla Referencial de Honorarios, la cual establece parámetros para prestaciones de servicios profesionales del trabajador social (CFESS, 2005). 
Por otra parte, este estudio evidencia que el vínculo laboral prevaleciente entre los trabajadores sociales y las instituciones en las que prestan servicios, tiende a ser de tipo honorarios. Un $44 \%$ de la muestra se encuentra en esta situación, le sigue en orden de importancia la contratación a plazo fijo con posibilidad de renovación en el tiempo, con un $22 \%$ y luego muy cercano el contrato de planta con un $20 \%$ de los encuestados. Si se suman los vínculos laborales: sin contrato, honorarios y de a plazo fijo sin claridad de renovación en el tiempo, tenemos que un $58 \%$ de los encuestados presentan vulnerabilidad en el vínculo laboral con la institución, sin contar que el $44 \%$ de ellos, no recibe ninguna protección de salud y jubilación por parte del empleador, quedando a la elección individual la cotización por este orden.

Frente a la precariedad y vulnerabilidad anteriormente mostrada, especialmente en lo que refiere al salario recibido y al tipo de contrato que prevalece entre los encuestados, es relevante que frente a la pregunta por la percepción de las condiciones laborales en las que se desenvuelven, el $38 \%$ las considere buenas, lo que sumado a quienes las perciben muy buenas, resulta que un $54 \%$ de los encuestados se sienten -al menos- bien o satisfechos con sus actuales condiciones laborales. En contraposición con el 20\% que las considera de regulares a malas, siendo esta última percepción un $6 \%$. Por otra parte, un $26 \%$ de quienes respondieron el cuestionario, no considera su situación contractual ni buena ni mala, porcentaje no menor, que puede ser interpretado como condiciones aceptables en los tiempos actuales. Una posible interpretación de este fenómeno es que las condiciones laborales precarias -sueldos bajos, precariedad de contrato- son subestimadas a la hora de ser conjugadas con la percepción del trabajo realizado al interior de la institución y con los destinatarios a los que dirigen sus acciones. Asimismo, la observación de las condiciones laborales precarias y de incertidumbre de la generalidad de los profesionales, incluso de otras áreas, generaría en ellos cierta naturalización del fenómeno.

Asimismo, un 54\% de los encuestados se encuentra buscando más empleo, lo que se relacionaría con los bajos ingresos recibidos y con que el salario del $50 \%$ de quienes respondieron el cuestionario, constituye en el principal o único ingreso económico familiar. Este indicador se transforma en la vía para suplir las carencias tanto del nivel de ingresos, como del vínculo laboral. No obstante, se debe 
considerar que la pluriempleabilidad es un fenómeno que ha tomado fuerza en las últimas décadas, y que si bien permite complementar el salario principal, la mayoría de las veces se realiza en condiciones de extrema precariedad para el trabajador, sin garantías de seguridad social, lo que, como se dijo anteriormente, puede afectar la calidad de la intervención profesional desarrollada.

Que el 54\% de los encuestados se encuentre buscando otro trabajo podría constituirse en una contradicción respecto de la favorable opinión de las condiciones laborales en que se desenvuelven. Es decir, ¿sería ello un encubrimiento de una negativa percepción del trabajo actual?

\section{Participación Colectiva}

Los datos muestran que el 22\% de los encuestados, participan en el Colegio de Asistentes Sociales, instancia que puede canalizar las demandas de sus asociados. El colegio de Asistentes Sociales en Chile es de larga data -en los años 50 fue creado- y en esta historia ha tenido un rol que ha variado según las circunstancias políticas del momento. Nació como una instancia significativa para las visitadoras sociales para vincularse, capacitarse y defender elementos propios de la profesión; durante la dictadura militar, el colegio es intervenido, evitando durante mucho tiempo la elección democrática de los cargos. A fines de la dictadura militar, se logra realizar elecciones democráticas y se comienzan a realizar acciones dirigidas a recuperar los derechos perdidos por la profesión durante el gobierno dictatorial, como por ejemplo, el rango universitario ${ }^{12}$. Sin embargo, las acciones realizadas por el Colegio profesional no han generado un fenómeno de adhesión significativa por parte de los profesionales.

Una posible explicación de esta baja participación, podría ser la crisis que están sufriendo -desde ya bastante tiempo- las instancias

12 "El 10 de marzo de 1990, en el último día de su mandato, la dictadura propinó un nuevo golpe al trabajo social: dicta la Ley Orgánica Constitucional de Enseñanza (LOCE) y lo excluye de las 12 carreras que a partir de ese momento tendrán carácter de licenciaturas universitarias. Esto significa situar la carrera en un nivel técnico que puede ser impartido por institutos profesionales (...) Esa situación académica ambivalente y sin fronteras precisas entre el nivel técnico y el universitario se mantuvo vigente durante 16 años." (Ruz y Hernández 2007:123) 
colectivas de representación, vinculadas al quehacer político, o las medidas que incorporan en el discurso la importancia de lo colectivo para la resolución de problemas. En ese sentido es conveniente situar el discurso de los encuestados referido al bajo interés por incorporarse al Colegio profesional. Dicho discurso alude al escaso tiempo con el que cuentan por las largas jornadas de trabajo y también a la escasa información acerca de los beneficios de esta incorporación. ¿No resulta contradictorio que los valores y principios que guían al trabajador social en su espacio laboral y frente a sus destinatarios, sean aquellos vinculados a la defensa de los derechos en todos sus niveles, la justicia social, la organización social o el desarrollo comunitario y éstos no se vinculen a las instancias para la defensa de su condición?

Lo anterior resulta preocupante, en la media que las mejoras laborales difícilmente se logran por acciones individuales y menos por iniciativa del empleador. La capacidad crítica pierde su fuerza sin una colectividad que le de sustentabilidad y organicidad. Así la preocupación por lo social implica también considerarse dentro de lo social.

\section{Conclusiones}

El presente estudio muestra vagamente algunos aspectos que han ido configurando las actuales condiciones laborales de los trabajadores sociales. Ello no sitúa en la necesidad de realizar otros estudios en la misma línea con el fin de avanzar en un análisis de mayor complejidad.

Partimos asumiendo algunas premisas: el mundo del trabajo y las condiciones en que se desenvuelve el ejercicio de cada profesional, nos puede iluminar algunos de los condicionamientos que existen cotidianamente para operacionalizar los valores y principios éticos que guían la profesión, como asimismo los desafíos a los que se está expuesto como categoría profesional. Es decir, las particularidades del ejercicio profesional, los resultados de la intervención son tensionadas por la condición de trabajador asalariado.

Sobre este supuesto y a partir de los datos encontrados en el estudio podemos concluir que:

La feminización de esta profesión es una característica que no se ha 
modificado a pesar del ingreso de hombres a la profesión a partir de los años 60' en Chile. Sin embargo, en la actualidad esta feminización dejó atrás su asociación originaria a aquellas características naturales con que se vinculaba "lo femenino" en los años '20, características personales que iban más allá de la preparación educacional.

En el origen de la primera Escuela de Servicio Social de Chile -vinculada al hacer médico y laico- se esperaba que sus estudiantes o futuro "personal especializado" desarrollara ciertas destrezas y características. Las mujeres, históricamente, han quedado excluidas de determinados empleos considerados masculinos por el tipo de competencias que se requieren, las que culturalmente se han definido como "masculinas" o "femeninas". Por ello, se ha promovido el ingreso de mujeres al mercado de trabajo en profesiones acordes a su "naturaleza". Es decir, profesiones donde se desarrollen tareas vinculadas a lo emotivo, lo particular, lo subjetivo, el cuidado y protección maternal, en ello las profesiones administrativas, educativas y asistenciales parecen ser áreas donde las mujeres tenderían a ingresar.

Por otro lado, los hombres son vinculados con profesiones científicas y técnicas, donde ponen en juego el conocimiento, el saber, la dirección, el ejercicio del poder o el ámbito político, por lo que lo racional, lo universal, lo objetivo tienden a ser las destrezas "naturales" masculinas que se requieren especialmente en la dirección o asesoramientos en el ámbito estatal, y en el manejo de grandes empresas o sistemas.

Los motivos para ingresar a estudiar esta profesión poseen rasgos que incluyen elementos de vocación y ayuda, lo que no se limita sólo a una especie de laicización de lo que históricamente se entendió dentro del contexto de caridad ${ }^{13}$, sino que incorpora nociones como justicia social ${ }^{14}$ y autonomía.

13 Es importante establecer distinciones conceptuales acerca de la caridad y la filantropía, con el fin de evitar lógicas homogenizantes. Caridad remite a la acción cristiana -la limosna- y filantropía es la superación de esta, una ayuda más continua. Ambas serían superadas a través de la profesionalización con un carácter científico y no moral. Ver: (Aylwin, Forttes y Matus 2004).

14 Recordemos que las últimas definiciones consensuadas internacionalmente acerca de la profesión de Trabajo Social, asumen como uno de sus principios la justicia social. 
Las condiciones laborales en que se desenvuelven los Trabajadores Sociales encuestados, muestra ciertos rasgos de precariedad, en tanto los contratos de trabajo se han vuelto inestables y los derechos sociales se han restringido, especialmente por prevalecer un contrato de tipo honorarios, a lo que se anexa el bajo nivel de remuneraciones recibidas. Un factor que no es posible dimensionar en este primer acercamiento, es el impacto del pluriempleo en el quehacer profesional.

Un aspecto invisibilizado en este primer sondeo es la desigualdad de género que cruza transversalmente a la profesión de Trabajo Social como a las diversas profesiones y la sociedad chilena en general. Es deducible que este elemento se presenta en la profesión, y que trae consecuencias sociales desiguales el pertenecer a uno $\mathrm{u}$ otro género, afectando negativamente a las mujeres. Se habla de la segunda jornada de trabajo para las mujeres, en que ésta desarrolla labores de casa, pero si ellas se encuentran llevando en paralelo dos empleos, se les suma una tercera jornada, ligada a los cuidados del hogar.

Recientes estudios en Chile, han sostenido la necesidad de profesionalizar las intervenciones que realizan las instituciones públicas -como las municipalidades- que se enfrentan directamente con una mayor "complejidad social", donde los índices de pobreza y de desarrollo humano es solo una parte de la manifestación de esta complejidad. Esta apuesta como forma de mejorar la calidad de la gestión involucra la actualización y la capacidad de innovación. Donde la profesionalización implica la permanente capacitación, situación que según los datos del presente estudio, está siendo puesta en marcha a pesar de los costos económicos en que deben incurrir los profesionales. Sin embargo, esta mayor capacitación no asegura que las precarias condiciones laborales en que se desempeñan los profesionales, sean modificadas institucionalmente o minimizadas por los mismos trabajadores sociales.

Por otro lado, es preciso mencionar que la precariedad de contratos unida a las bajas remuneraciones, fomentan la alta rotación de profesionales en búsqueda de mejores perspectivas laborales, afectando con ello la gestión de largo plazo de las intervenciones. Este estudio evidencia que más del 50\% de los encuestados busca cambiar de empleo a pesar de sentirse valorado por la institución. La 
conjunción de todos los factores mencionados debe iluminar sobre las implicancias que ello puede tener en los niveles de rendimiento profesional de estos trabajadores. Es preciso manifestar la relación directa entre los programas y planes que implementan los trabajadores sociales y las complejas manifestaciones de la cuestión social. Ante ello, la preocupación que surge es acerca de las posibilidades que tienen de realizar intervenciones que no se conviertan en una mera acción repetitiva y acrítica cuando, por ejemplo, sostienen dos trabajos o los salarios recibidos apenas superan la línea de la pobreza, afectándose así los resultados de la intervención social.

La profesión se encuentra inmersa en un movimiento que permite la continuidad de la situación actual, por ejemplo, en relación a las condiciones laborales presentes y futuras de los colegas, pero también está en sus manos generar posibilidades de transformación de tal escenario.

No es posible que frente a los actuales desafíos de la profesión en Chile se actúe de forma individual, ya que el panorama no es muy esperanzador por esa vía. La profesión no sólo requiere de intenciones personales, sino además de un trabajo colectivo para asumir los desafíos y generar los cambios requeridos para un mejoramiento de las condiciones en que se desenvuelve, lo que sin duda tendrá consecuencias positivas en los resultados de su quehacer profesional.

\section{Referencias bibliográficas}

ANTUNES, R. (1999).

ARENDT, H. (1998).
BARRÍA, J. (2002).

¿Adiós al Trabajo? Ensayo sobre la metamorfosis y el rol central del mundo del trabajo. Buenos Aires: Editorial Antídoto.

La condición humana. Barcelona: Paidós.

Síndrome de Burnout en asistentes sociales del Servicio Nacional de Menores de la Región Metropolitana de Chile. En Psiquiatría.com, 6 (4). Recuperado el 20 de septiembre 2008, de http://www.psiquiatria.com/articulos/psiqsocial/11687/

CADEMARTORI, F. CAMPOS, J. Y SEIFFER, T. (2007).

Condiciones de trabajo de los trabajadores sociales. Hacia un proyecto profesional crítico. Buenos Aires: Espacio. 
CONSELHO FEDERAL DE SERVIÇO SOCIAL (2005).

Assistentes Sociais no Brasil. Elementos para o estudo do perfil profissional. Brasilia: AUTOR

DEPOLO, S. (2007). Exclusión Social en el Mercado Laboral Chileno. Elementos para analizar el caso de los profesionales altamente calificados. En Revista Mad, 16. Recuperado el 14 de septiembre de 2008. De http://www.revistamad.uchile.cl/16/ depolo_02.pdf

GUERRA, Y. (2000). La crisis contemporánea y los impactos en la instrumentalización del trabajo social. En Boletín Electrónico Sura, 45. Recuperado el 15 de septiembre de 2008. De http://www.ts.ucr.ac.cr/ suradoc.htm

HENRIQUEZ, H. y RIQUELME, V. (2006). Lejos del trabajo decente: el empleo desprotegido en Chile. [Versión electrónical. En Cuadernos de investigación, 30. Recuperado el 5 de septiembre de 2008. De http://www. dt.gob.cl/documentacion/1612/articles-94001_ recurso_1.pdf

IAMAMOTO, M. (2007). Serviço Social em Tempo de Capital Fetiche. Capital financiero, trabalho e questao social. Sao Paulo: Cortez Editora.

ILLANES, M. A. (2007). C Cuerpo y Sangre de la Política. La Construcción Histórica de las Visitadoras Sociales (1887-1940). Santiago de Chile: LOM Ediciones.

MATUS, T. y QUEZADA, M. (2007). Los desafíos de trabajar la pobreza con calidad: análisis de la relación complejidad versus condiciones de calidad en el ámbito social municipal de las 52 comunas de la Región Metropolitana. En La reforma municipal en la mira identificando los municipios prioritarios en la Región Metropolitana: Complejidad comunal versus condiciones para la calidad de la gestión municipal (cap.4). Recuperado el 10 de septiembre de 2008. De http://www.expansiva.cl/media/archivos/20071109111558.pdf

MATUS, T, AYLWIN, N. y FORTTES, A. (2004). La Reinvención de la Memoria. Indagación sobre el proceso de profesionalización del Trabajo Social Chileno 1925-1965. Santiago, Chile: Pontificia Universidad Católica de Chile. 
SÁnCHEZ, ADOLFO. Filosofía da Praxis. Sao Paulo: Coleçao Pensamento Social Latino-Americano. Clacso, 2007.

PALAZÓN, M. (2007). L L L filosofía de la praxis según Adolfo Sánchez. Recuperado el 10 de septiembre de 2008. De http://bibliotecavirtual.clacso.org.ar/ar/libros/campus/ marxis/P2C5Mayoral.pdf

STIGLITZ, J. (2004). El Consenso Post-Consenso de Washington. Recuperado el 10 de septiembre de 2008. De http:// www0.gsb.columbia.edu/ipd/pub/Stiglitz PWCC_SPA.pdf

RUZ, O. y HERNÁNDEZ, J. (2007). El Trabajo Social en Chile. En Deslauriers, J. y Hurtubise, Y, El Trabajo Social Internacional. Elementos de comparación (pp.126-127). Buenos Aires: Lumen-Humanitas. 
